\title{
Windowed Equivalence Principle for Open Surfaces
}

\author{
Manouchehr Takrimi ${ }^{1,2}$ and Levent Gürel ${ }^{1,2}$ \\ ${ }^{1}$ Department of Electrical and Electronics Engineering, Bilkent University, Ankara, TR-06800, Turkey \\ ${ }^{2}$ Computational Electromagnetics Research Center (BiLCEM), Ankara, TR-06800, Turkey \\ takrimi@ee.bilkent.edu.tr
}

\begin{abstract}
We introduce a modified current expansion scheme over open surfaces based on the equivalence theorem, which employs closed surfaces, in principle. Weighting the expansion coefficients with a suitable window function compensates for the computed field errors that occur because of the open surfaces. Numerical simulations demonstrate that the equivalent surface currents expanded with low-ordered basis functions on an open surface and weighted by suitable functions can be used to obtain the correct electromagnetic fields in a limited volume near the surface.
\end{abstract}

\section{INTRODUCTION}

The surface equivalence theorem is a principle by which actual sources, such as antennas, are replaced by equivalent sources. The theorem was introduced by Schelkunoff [1] and is based on the uniqueness theorem, which states [2] that "a field in a region is uniquely specified by the sources within the region plus a proper combination of the tangential components of the electric/magnetic fields over the boundary."

Invoking the uniqueness principle, fields inside an imaginary or physical closed surface that encloses a domain may be considered to be generated by suitable equivalent source distributions satisfying the boundary conditions over the closed surface. The associated equivalence source distributions are not unique, so we prefer to use Love's equivalence principle, which is based on both electric and magnetic currents. The degree of accuracy depends on the knowledge of the tangential components of the fields over the above noted closed surface.

We wish to develop a method that will yield the original fields inside a limited volume near an open surface. Knowing that the aforementioned theorems are based on closed surfaces, we commence by establishing an equivalent state using a closed surface, and later, we proceed to open that surface by introducing a "windowed equivalence principle" (WEP).

Before we introduce an equivalent state, we should first define the corresponding original state. An actual radiating source, which is represented electrically by the current densities $\boldsymbol{J}$ and $\boldsymbol{M}$ are shown in Fig. 1(a). This original source radiates the fields $\boldsymbol{E}$ and $\boldsymbol{H}$ everywhere. The volume within $S$ is denoted by $V_{i}$, and outside of $S$ it is denoted by $V_{o}$. An equivalent problem for Fig. 1(a) is shown in Fig. 1(b). Here we assume that $V_{i}$ is the "region of interest" and there exist the original fields $\boldsymbol{E}, \boldsymbol{H}$ inside $S$, and some other Maxwellian fields $\boldsymbol{E}_{\text {out }}, \boldsymbol{H}_{\text {out }}$ outside of $S$. For these fields to exist within and outside of $S$, they must satisfy the boundary conditions of the tangential electric and magnetic field components. Thus, on the imaginary surface $S$, there must exist equivalent sources such that:

$$
\left\{\begin{array}{c}
\boldsymbol{J}_{s}=\hat{\boldsymbol{n}} \times\left(\boldsymbol{H}_{\mathrm{out}}-\boldsymbol{H}\right) \\
\boldsymbol{M}_{s}=-\hat{\boldsymbol{n}} \times\left(\boldsymbol{E}_{\mathrm{out}}-\boldsymbol{E}\right) .
\end{array}\right.
$$

These equivalent currents produce the original fields only inside of $S$. Since $V_{o}$ is not the region of interest, the fields ( $\left.\boldsymbol{E}_{\text {out }}, \boldsymbol{H}_{\text {out }}\right)$ outside of $S$, can be assumed to be zero. Then, the equivalent current densities reduce to

$$
\left\{\begin{aligned}
\boldsymbol{J}_{s} & =-\hat{\boldsymbol{n}} \times \boldsymbol{H} \\
\boldsymbol{M}_{s} & =\hat{\boldsymbol{n}} \times \boldsymbol{E} .
\end{aligned}\right.
$$

In order to regenerate the original $\boldsymbol{E}$ and $\boldsymbol{H}$ fields inside $S$, we need to compute the equivalent currents all over the surface, no matter how large it is. For electrically large problems with large surfaces, it takes a huge amount of computer resource to calculate and store the equivalent surface currents.

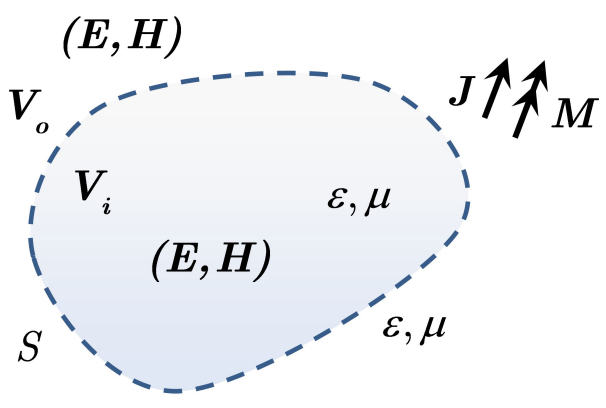

(a)

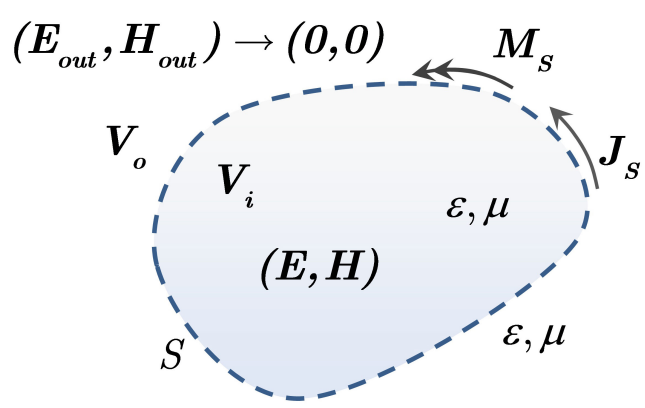

(b)

Fig. 1. Surface equivalence theorem. (a) Original problem. (b) Equivalent problem. 
The problem becomes even more difficult when the "region of interest" is not bounded with a closed surface. Theoretically, we should not use the equivalence principle over open surfaces; and if we do, we must expect some computational errors. Even though such errors are unavoidable, we may define a limited (as opposed to unbounded) region of interest, where the maximum error can be kept under a predetermined level. This is useful in some practical problems, where the original fields inside a limited volume in a specific region need to be computed from the equivalent currents .

\section{Proposed Method}

This paper proposes to apply well-known window functions, such as "generalized cosine windows" (which are commonly used in signal processing applications [3] and in other branches of science [4]) to overcome the errors introduced by truncating equivalent surface currents at the edges of open surfaces. A higher-order generalized cosine window function is defined by [5]

$$
\operatorname{win}(\rho)=\sum_{k=0}^{K} a_{k} \cos \left(\frac{2 k \pi \rho}{R}\right),
$$

where $K$ is the order of the function and $2 R$ is the effective domain of the function. Choosing $K=2$ and the coefficients $a_{0}=-a_{1}=1 / 2$ gives us the Hanning (raised cosine) window, which will be used through the rest of this paper. Values of the Hanning window are tapered down to zero at the boundaries of the effective domain. The reason for using a window function is to mitigate the sudden changes of currents due to their truncation at the boundaries. Obviously for numerical computation of the radiated fields resulting from truncated currents and weighted by the window function, we need to discretize the surface using small planar triangles and employ the Rao-Wilton-Glisson (RWG) functions [6] to expand the known equivalent surface current densities $\boldsymbol{J}_{s}$ and $\boldsymbol{M}_{s}$. As noted above, while the goal is to reduce the computational errors arising from the use of open surfaces instead of closed ones, we expect also the other errors due to the discretization, singularity near equivalent currents, mesh size, computation and integration accuracy, and more. Surface shape, volume width and depth, choice of the window function, and the specifics of the incident field are among the key parameters determining the accuracy of results.

\section{NUMERICAL RESULTS}

To hypothesize what might occur when we use the equivalent surface currents defined in Eq. (2) over an open surface, we use a horizontal dipole radiating at $300 \mathrm{MHz}(\lambda=1 \mathrm{~m})$ as a source. The dipole is positioned $1 \mathrm{~m}$ above an imaginary planar and circular surface of radius $R=10 \mathrm{~m}$, and the fields underneath the surface are computed. Figure 2 illustrates the configuration of the simulation environment. We use the following metric as a figure of merit to calculate the error incurred by truncating the currents at the boundaries of an open surface with respect to the original incident field coming

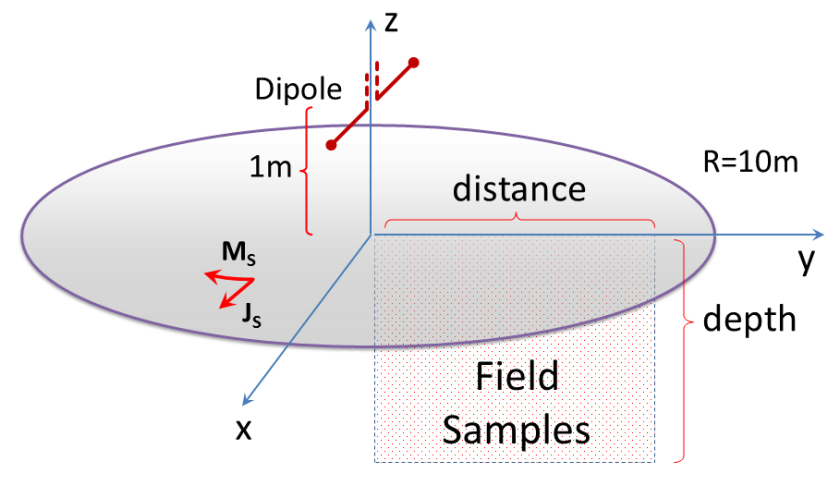

Fig. 2. Field sampling points underneath an imaginary planar and circular shape of radius $R$ illuminated by a horizental dipole.

from the dipole:

$$
\text { Field Error(\%) }=100 \times \frac{\left\|\boldsymbol{E}_{\text {computed }}-\boldsymbol{E}_{\text {incident }}\right\|}{\left\|\boldsymbol{E}_{\text {incident }}\right\|} .
$$

Figure 3 illustrates the percentage of error as a function of horizontal distance from the source and vertical distance (depth) from the surface both in meters. The contours in Fig. 3 reveal that the error increases rapidly with the distance from the surface and decreases in a periodic manner moving along the surface. This situation is mainly due to the phase errors caused by the truncated currents at the edges. Applying $70 \%$ partial windowing (i.e., using the window function for a radius larger than $30 \%$ of $\mathrm{R}$ ) on coefficients obtained from the RWG expansion effectively decreases the error to below $1 \%$ for almost entire area, as shown in Fig. 4. Repeating the same procedure for a vertical dipole yields similar results. Figure 5 shows the field error for a vertical dipole at the same position. Using the same window function, this time

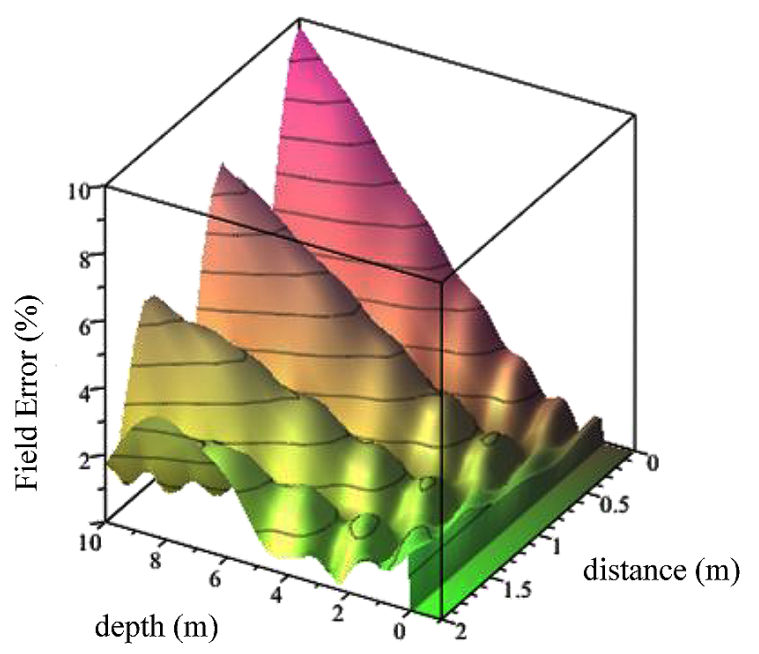

Fig. 3. Percentage of field error between computed electric field and incident electric field for a horizontal electric dipole without windowing. 
by an amount of $60 \%$, brings the field error down to about $1 \%$. A wide range of simulations with different scenarios were carried out to confirm the usefulness of the proposed technique. The simulations show that $1 \%$ (or less) error can be obtained for depths of $R / 5$ to $R$, as evident from Figs. 4 and 6 . Furthermore, errors below $1 \%$ can be achieved at depths less than $R / 5$ for distances (from the origin) less than $R / 5$. All of these observations depend on the proper choice of the window function.

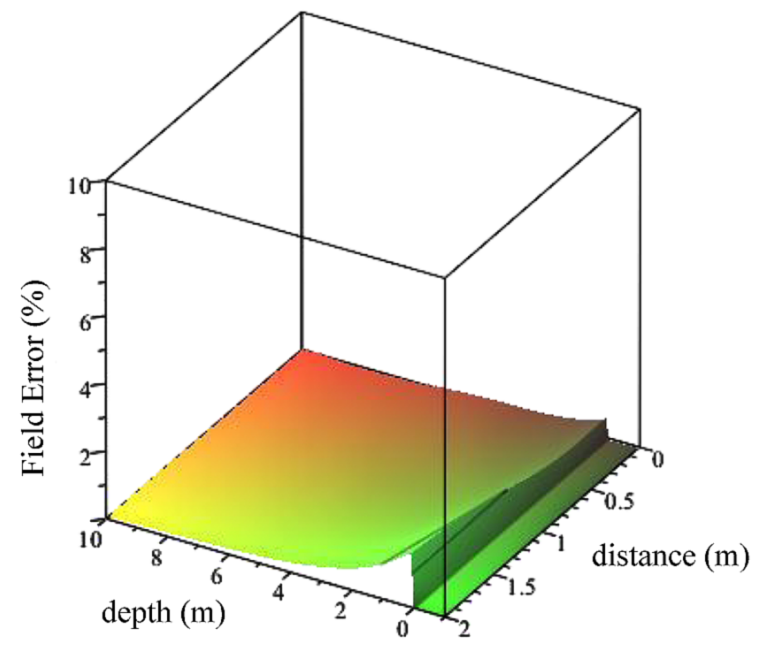

Fig. 4. The effect of applying $70 \%$ partial windowing to reduce the field errors shown on Fig. 3.

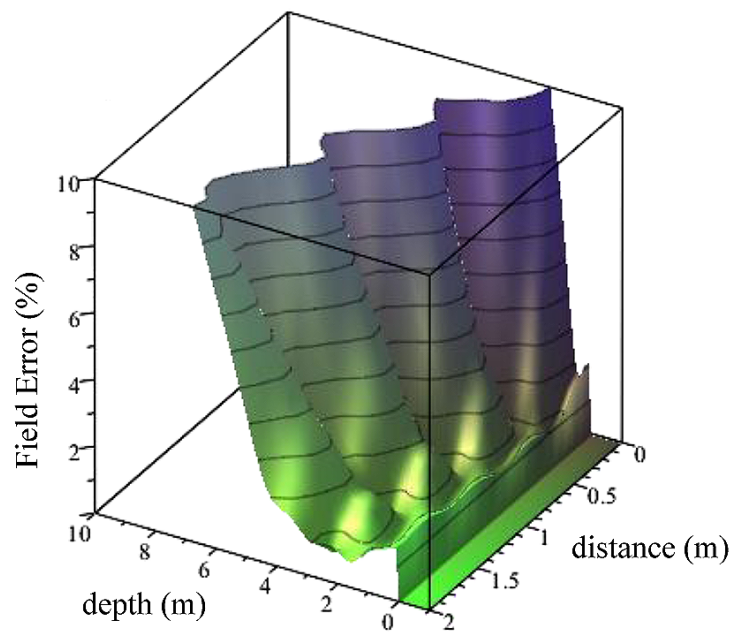

Fig. 5. Percentage of field error between computed electric field and incident electric field for a vertical electric dipole without windowing.

\section{CONCLUSIONS}

This paper proposes a new technique, namely, WEP, to reduce the errors originating from using equivalent current sources over open surfaces. By adjusting window parameters,

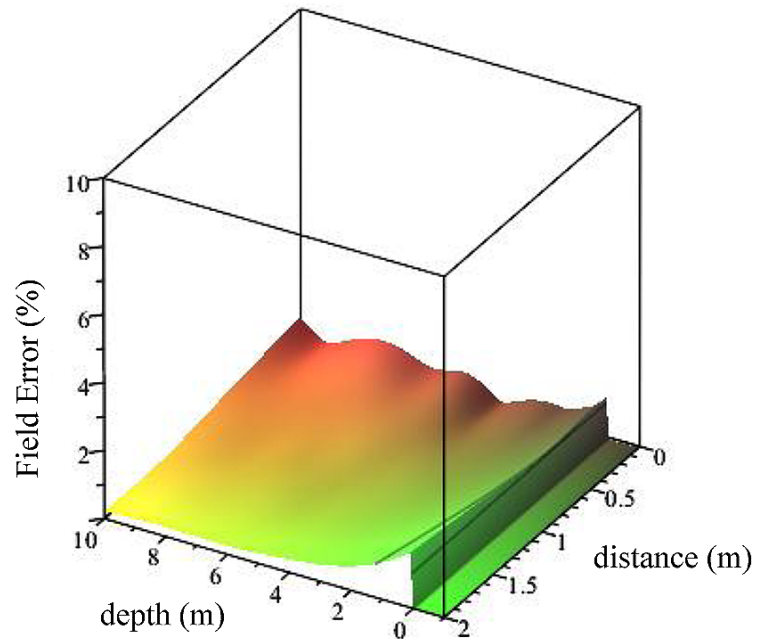

Fig. 6. The effect of applying $60 \%$ partial windowing to reduce the errors shown in Fig. 5.

optimum accuracy (minimum error) can be achieved based on the specifics of the source and the surface. Errors below 1\% are achieved in relatively large volumes using WEP.

\section{ACKNOWLEDGMENT}

This work was supported by the Scientific and Technical Research Council of Turkey (TUBITAK) under Research Grants 110E268 and 111E203, by Schlumberger-Doll Research (SDR), and by contracts from ASELSAN, Turkish Aerospace Industries (TAI), and the Undersecretariat for Defense Industries (SSM).

\section{REFERENCES}

[1] S. Schelkunoff, "Some equivalence theorems of electromagnetics and their application to radiation problems," Bell Syst. Tech. J., vol. 15, no. 1, pp. 92-112, 1936.

[2] C. A. Balanis, Advanced Engineering Electromagnetics. New York: Wiley, 1989.

[3] J. Allen, D. Berkley, and J. Blauert, "Multimicrophone signal-processing technique to remove room reverberation from speech signals," J. Acoust. Soc. Amer., vol. 62, pp. 912-915, 1977.

[4] J. Schmit and K. Creath, "Window function influence on phase error in phase-shifting algorithms," Appl. Opt., vol. 35, no. 28, pp. 5642-5649, 1996.

[5] [Online]. Available: http://www.mathworks.com/help/signal/ref/hann.html

[6] S. Rao, D. Wilton, and A. Glisson, "Electromagnetic scattering by surfaces of arbitrary shape," IEEE Trans. Antennas Propag., vol. 30, no. 3, pp. 409-418, May 1982. 